\title{
Optimizing the mechanical device of a mono-axial sun tracking mechanism
}

\author{
Cătălin Alexandru*
}

Transilvania University of Brașov, Product Design, Mechatronics and Environment Department, 500036 Braşov, Romania

\begin{abstract}
The paper deals with the dynamic optimization of the mechanical device for a mono-axial sun tracking system, with application for photovoltaic and solar-thermal systems, which convert the solar radiation in electrical or respectively thermal energy. The actuating of the mono-axial tracking mechanism is carried out by using a linear actuator, the optimization study intending to determine the optimal configuration of the mechanism with the aim to minimize the motor force developed by actuator in order to perform the imposed step-by-step tracking algorithm (motion law). The design variables in the optimal design process are represented by the global coordinates of the connection points of the linear actuator to the adjacent parts (the solar panel and the fixed support). The optimization study is conducted in virtual prototyping environment.
\end{abstract}

\section{Introduction}

In order to increase the efficiency of the solar panels (photovoltaic and solar thermal panels/collectors), a commonly used solution consists of equipping them with tracking systems (also called solar trackers), which allow the panel/collector to follow the trajectory of the sun on the celestial vault, so as to capture a greater amount of incident solar radiation [1-4]. By using such systems, the energetic efficiency of the solar panels/collectors can be significantly increased, in correlation with the specific geographic and climatic conditions [5-7].

The active tracking mechanisms of the solar panels are mechatronic systems, which integrate mechanical and electronic components. Generally, the optimization of the dynamic behavior of the tracking systems addresses two aspects (corresponding to the two main components of the mechatronic product):

- the geometric optimization of the mechanical structure (device), in order to minimize the driving forces developed by the actuators for performing the required motion (tracking) laws;

- the optimization of the control system in order to reduce the transient period, and minimize the tracking errors.

The both optimization ways aim, in fact, to reduce the energy consumption necessary to achieve the orientation, with a beneficial effect on the energy efficiency of PV tracking system. For the present work, the study is focused on the first optimization way (optimal design of the mechanical device). The dynamic model of the solar tracker was designed in mechatronic concept, by using MBS (Multi-Body Systems) and DFC (Design for Control) software solutions (namely, ADAMS and EASY5 of MSC.Software).

\section{Setting the optimization problem}

The use of a virtual prototyping platform offers the possibility to easily optimize the behavior of the mechanical \& mechatronic systems by quickly testing a multitude of design variants (in terms of location, orientation and characteristics of the specific elements, mass distribution and so on), at reduced costs compared to the optimization process based on building $\&$ testing of physical (experimental) prototypes [8-10].

The optimization of the solar panel tracking systems from the point of view of the geometrical configuration of the mechanical device is based on the following stages: parameterizing the MBS virtual model; modeling the design variables, constraints and objectives; carrying out parametric design studies to identify the main design variables (with significant influence on the dynamic behavior of the tracking system, which is expressed by the design objectives); optimizing the virtual model by identifying the optimal values of the main design variables that assure, by case, the minimization or maximization of the design objective functions.

In this study, the goal of the dynamic optimization of the solar panel tracking systems is to minimize the motor force developed by the driving actuator, which in fact leads to a reduction in energy consumption (demand) to achieve the orientation. The case study is developed for a mono-axial tracking system, with one degree of freedom (DOF), corresponding to the diurnal movement of the solar panel, while the elevation is maintained at a fixed position. The solar panel is operated by means of a linear actuator disposed between the panel frame and the fixed support, the step-by-step motion law being modeled so as to achieve a minimum number of actuations (for energy efficiency considerations).

The virtual model of the mechanical device of the mono-axial solar tracker, which was developed by using the MBS software solution ADAMS, is shown in figure 1.

* Corresponding author: calex@unitbv.ro

(C) The Authors, published by EDP Sciences. This is an open access article distributed under the terms of the Creative Commons Attribution License 4.0 (http://creativecommons.org/licenses/by/4.0/). 
On the other hand, the control system diagram block (figure 2) was developed by using a specific DFC (Design for Control) software solution (EASY5), the detailed description of the control device following to be presented in a future paper.

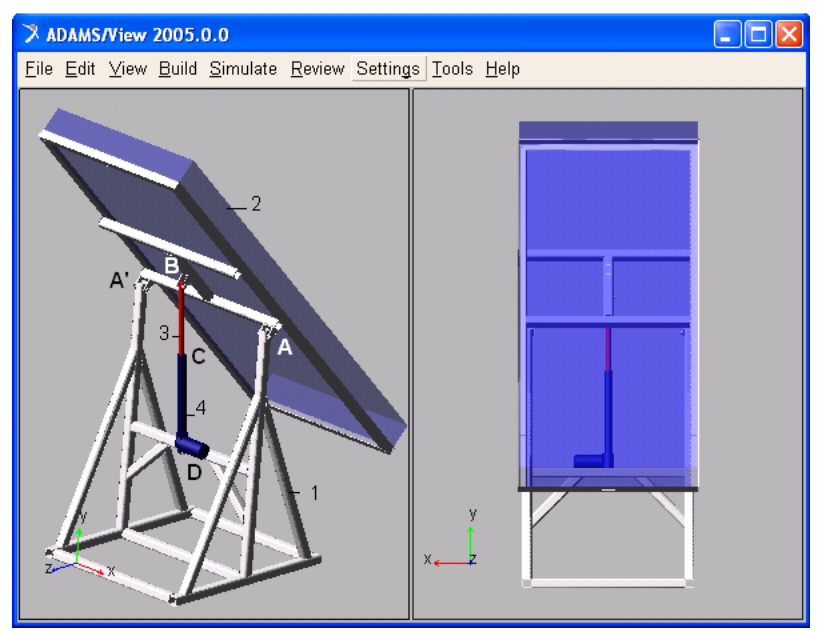

Fig. 1. The MBS model of the tracking mechanism.

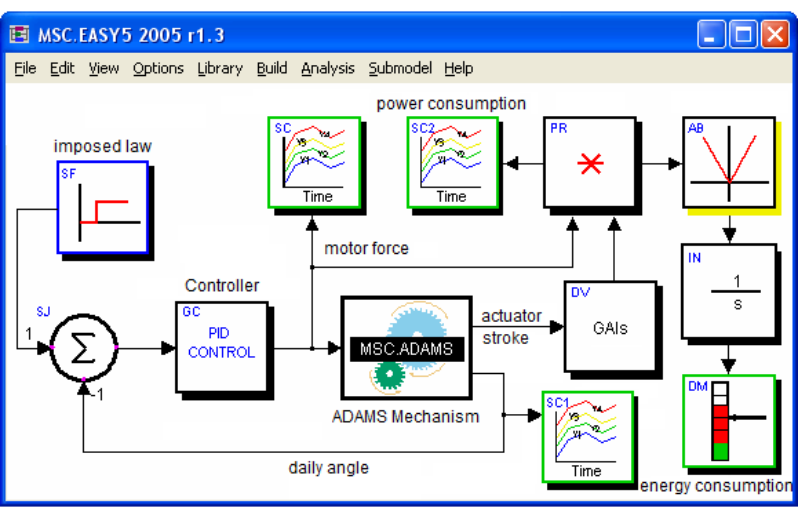

Fig. 2. The DFC model of the control system.

The controlled parameter (which is input in the control system model) is represented by the diurnal angle of the solar panel, while the motor force developed by the linear actuator defines the input in the mechanical device model. The communication between the mechanical \& control components is managed through the ADAMS/Controls interface.

For the present paper, the numerical simulations are made considering the data specific to the Braşov geographical area (latitude - 45.6333 North, longitude 25.5833 East), on the day of the summer solstice ( 21 of June, sunshine local time - 5.466, sunset local time 21.183). The step-by-step motion (tracking) program was designed in accordance with the algorithm presented in [5], as follows: the angular domain for the diurnal movement $-\beta^{*} \in\left[64^{\circ},-64^{\circ}\right]$, where $\beta^{*}=0^{\circ}$ corresponds to the solar noon position; the actuating timing (in local time): $9.319\left(\Delta \beta^{*}=14^{\circ}\right), 10.319\left(\Delta \beta^{*}=14^{\circ}\right), 11.239$ $\left(\Delta \beta^{*}=12^{\circ}\right), 12.089\left(\Delta \beta^{*}=12^{\circ}\right), 12.919\left(\Delta \beta^{*}=12^{\circ}\right), 13.719$ $\left(\Delta \beta^{*}=12^{\circ}\right), 14.549\left(\Delta \beta^{*}=12^{\circ}\right), 15.399\left(\Delta \beta^{*}=12^{\circ}\right), 16.319$ $\left(\Delta \beta^{*}=14^{\circ}\right), 17.319 \quad\left(\Delta \beta^{*}=14^{\circ}\right)$; return to the initial position (local time): 21.183 .
For these input data, the time history variations of the daily angle, which is measured in the revolute joint A-A' between panel and support, and the corresponding angular velocity at which the diurnal movement is performed are shown in figure 3. By the inverse kinematics of the tracking mechanism, the correlation between the position angle of the solar panel and the linear stroke in the driving actuator is established.
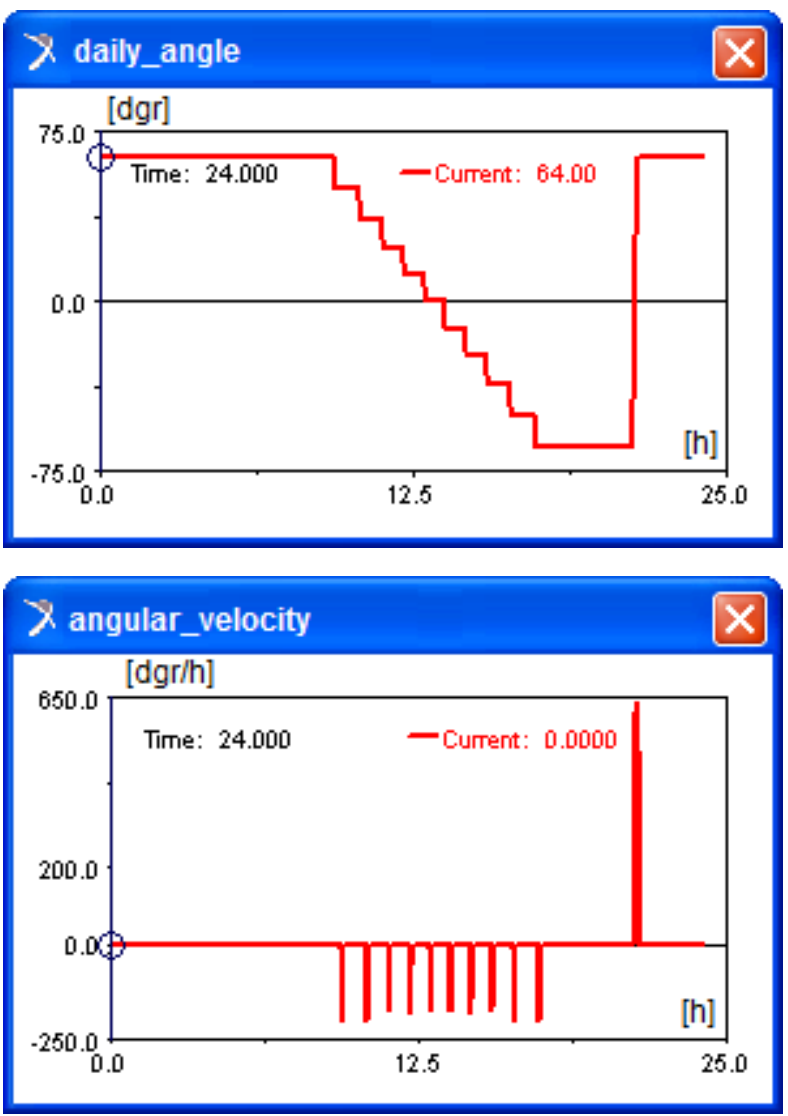

Fig. 3. The step-by-step tracking law.

The parameterization of the virtual model of the tracking system shown in figure 1 is based on the design points that define the structural scheme of the system, in this case the locations of the joints between bodies. For the optimization study, the connection points of the linear actuator to the adjacent elements (piston to panel frame $\mathrm{B}$, and cylinder to support - D) have been considered. The locations of the revolute joints between the panel frame and the fixed support (A - A ') are considered to be established on constructive criteria.

In this way, 6 design variables, which will control the model during the optimization, have been obtained, namely the global Cartesian coordinates (in the global reference frame $\mathrm{XYZ}$ ) of the design points $\mathrm{B}$ and $\mathrm{D}$ : DV $1 \rightarrow X_{\mathrm{B}}$, DV $2 \rightarrow \mathrm{Y}_{\mathrm{B}}, \mathrm{DV} \_3 \rightarrow \mathrm{Z}_{\mathrm{B}}$, DV $4 \rightarrow \mathrm{X}_{\mathrm{D}}$, DV_5 $\rightarrow Y_{D}$, and DV_6 $\rightarrow Z_{D}$. Each design variable is defined by an initial value, and a variation range relative to the initial value, which was set in view of maintaining the system within acceptable constructive limits.

In order to maintain the relative location and orientation in the translational joint between the two components of the actuator (cylinder and piston), the two 
joint coordinate systems have been parameterized using the following expressions: LOC_RELATIVE_TO $(\{0,0$, $0\}$, POINT_C) - the function sets the location of the coordinate systems that define the translational joint relative to the position of the design point $\mathrm{C}$; ORI_ALONG_AXIS (POINT_D, POINT_B, "Z") - the function sets the $Z$-axis orientation of the coordinate systems, representing the translational motion axis, in the direction defined by the design points $\mathrm{D}$ and $\mathrm{B}$.

On the other hand, a design constraint has been defined to control the value of the pressure angle measured between the force vector generated by the actuator and the linear velocity vector on the solar panel at point B. Theoretically, the tracking mechanism selflocks when the pressure angle reaches $90^{\circ}$, but practically, due to the friction, the self-locking occurs at values less than the theoretical boundary. For safety reasons, the maximum acceptable value of the pressure angle was set at $65^{\circ}$. In ADAMS, the sign of the measure defining a constraint has a special significance. For example, if a constraint is positive, then it is violated, or if it is negative, the constraint has not been violated. In these terms, the design constraint was defined by a measure that subtracts the maximum accepted value from the current value of the pressure angle.

\section{Results and conclusions}

The parametric design studies have been carried out to determine the sensitivity of the objective function (motor force) to the modification of the six design variables. Such a study is performed for each variable, by swapping it in the specific variation domain (value range), considering the other variables with the initial (standard) value. As result of these studies, the main design variables (with significant influence on the design objective) have been identified, namely DV_2, DV_3 and DV_6. The other design variables (DV_1, DV_4 and DV_5) are secondary variables (with small influence on the design objective), which will be neglected in the optimization process (they will be considered with the initial/standard values).

In the initial design, before optimization, the dynamic behaviour of the tracking system is defined by the results shown in figure 4 (the time history variations of the motor force, power \& energy consumption, and pressure angle). The optimization of the mechanical device was performed on the basis of the main variables, by seeking the optimal values of these variables in the considered value ranges (by simultaneously changing all the main design variables DV_2, DV_3 and DV_6). The optimal design, after optimization, is defined by the results shown in figure 5 .

The optimization leads to a significant reduction in power/energy consumption for performing the tracking, with minor changes in the geometric configuration of the mechanism. At the same time, the pressure angle remains within the required limits.
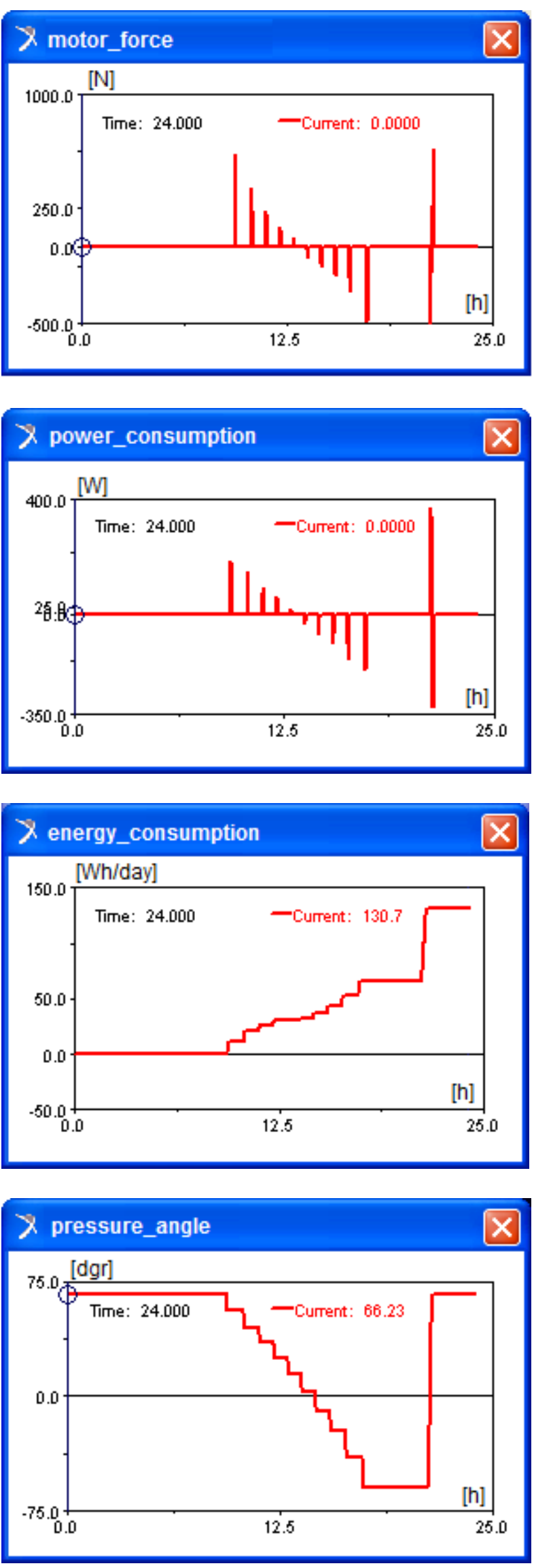

Fig. 4. Results specific to the initial design of the mechanism (before optimization). 

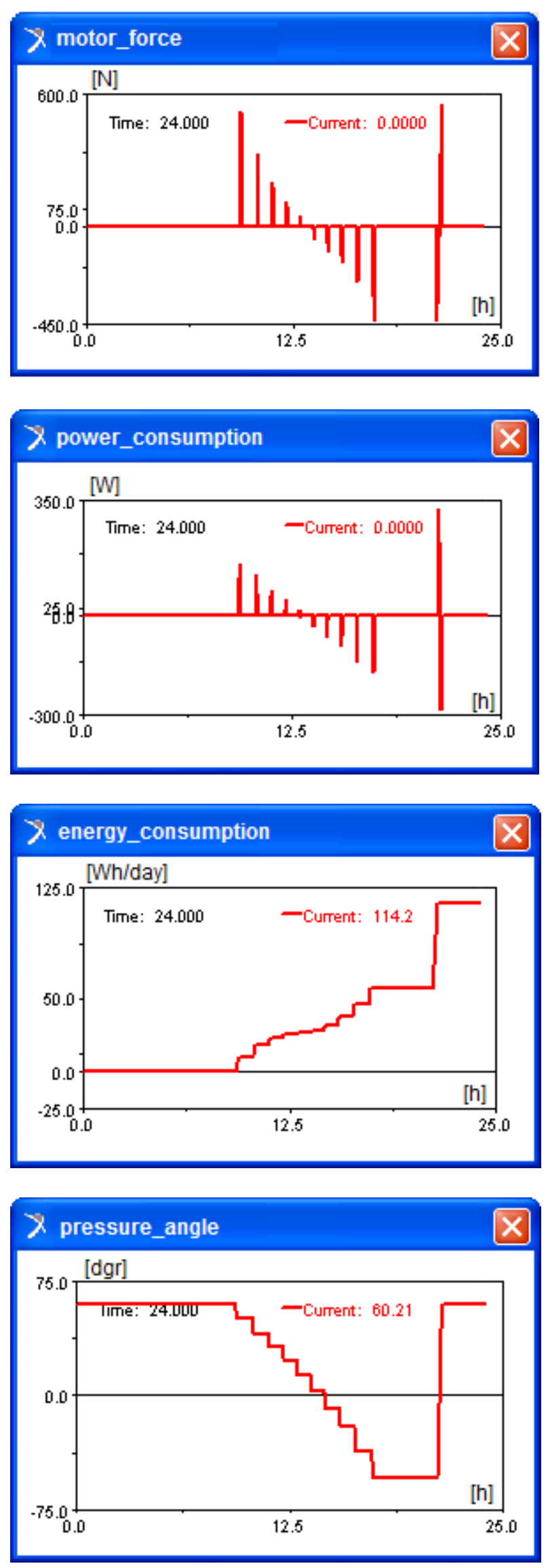

Fig. 5. Results specific to the optimal design of the mechanism (after optimization).
The application presented in this work is a suggestive example on the facilities offered by the virtual prototyping technique in the optimal design process of the mechatronic tracking systems of the solar panels. The virtual prototype of the solar tracker allows the assessment of various tracking strategies (in terms of angular motion domain, number of actuation steps, actuating timing) in order to increase the energy efficiency and reliability of the sun tracking mechanism (by maximizing the solar radiation input, minimizing the power/energy consumption to achieve orientation, minimizing the operating time of the driving actuators).

\section{References}

1. C. Alexandru, M. Comşiţ. The energy balance of the photovoltaic tracking systems using virtual prototyping platform. 5th IEEE International Conference on the European Electricity Market EEM, pp. 253-258 (2008)

2. C. Alexandru. The design and optimization of $a$ photovoltaic tracking mechanism. 2nd IEEE International Conference on Power Engineering, Energy and Electrical Drives POWERENG, pp. 436441 (2009)

3. D.A. Flores-Hernández, S. Palomino-Resendiz, N. Lozada-Castilloc, A. Luviano-Juárez. Mechatronic design and implementation of a two axes sun tracking photovoltaic system driven by a robotic sensor. Mechatronics, 47, pp. 148-159 (2017)

4. F.M. Hoffmann, R.F. Molz, J.V. Kothe, E.O.B. Nara, L. Pablo, C. Tedesco. Monthly profile analysis based on a two-axis solar tracker proposal for photovoltaic panels. Renewable Energy, 115, pp. $750-759$ (2018)

5. C. Alexandru, I.N. Tatu. Optimal design of the solar tracker used for a photovoltaic string. Journal of Renewable and Sustainable Energy, 5, pp. 1-16 (2013)

6. S. Seme, B. Stumberger, M. Hadziselimovic. A novel prediction algorithm for solar angles using second derivative of the energy for photovoltaic sun tracking purposes. Solar Energy, 137, pp. 201-211 (2016)

7. S.A. Sharaf Eldin, M.S. Abd-Elhady, H.A. Kandil. Feasibility of solar tracking systems for PV panels in hot and cold regions. Renewable Energy, 85, pp. 228233 (2016)

8. C. Alexandru, C. Pozna, C. Dynamic modeling and control of the windshield wiper mechanisms. WSEAS Transactions on Systems, 8, pp. 825-834 (2009)

9. C. Alexandru, P. Alexandru. The virtual prototype of a mechatronic suspension system with active force control. WSEAS Transactions on Systems, 9, pp. 927-936 (2010)

10. D. Tarniţă, M. Catană, D.N. Tarniță. Contributions on the modeling and simulation of the human knee joint with applications to the robotic structures. Mechanisms and Machine Science, 20, pp. 283-297 (2014) 